\title{
Development of twist in an emerging magnetic flux tube by poloidal field accretion
}

\author{
P. Chatterjee ${ }^{1}$, A. R. Choudhuri ${ }^{1,2}$, and K. Petrovay ${ }^{1,2,3}$ \\ 1 Department of Physics, Indian Institute of Science, Bangalore 560012, India \\ 2 Eötvös University, Department of Astronomy, Budapest, Pf. 32, 1518, Hungary \\ e-mail: K.Petrovay@astro.elte.hu \\ 3 Department of Applied Mathematics, University of Sheffield, Hicks Building, Hounsfield Road, Sheffield S3 7RH, UK
}

Received 24 October 2005 / Accepted 8 December 2005

\section{ABSTRACT}

Aims. Following an earlier proposal for the origin of twist in the magnetic fields of solar active regions, we model the penetration of a wrapped up background poloidal field into a toroidal magnetic flux tube rising through the solar convective zone.

Methods. The rise of the straight, cylindrical flux tube is followed by numerically solving the induction equation in a comoving Lagrangian frame, while an external poloidal magnetic field is assumed to be radially advected onto the tube with a speed corresponding to the rise velocity. Results. One prediction of our model is the existence of a ring of reverse current helicity on the periphery of active regions. On the other hand, the amplitude of the resulting twist depends sensitively on the assumed structure (diffuse vs. concentrated/intermittent) of the active region magnetic field right before its emergence, and on the assumed vertical profile of the poloidal field. Nevertheless, in the model with the most plausible choice of assumptions a mean twist comparable to the observations results.

Conclusions. Our results indicate that the contribution of this mechanism to the twist can be quite significant, and under favourable circumstances it can potentially account for most of the current helicity observed in active regions.

Key words. Sun: interior - Sun: magnetic fields - magnetohydrodynamics (MHD)

\section{Introduction}

The magnetic field of a typical sunspot is approximately vertical below the photosphere and then spreads radially at the photospheric level. However, even visual observations show that the penumbral structures of sunspots are often twisted, as first pointed out by Hale (1927) and Richardson (1941). Vector magnetogram measurements in the past decade have established that sunspot magnetic fields have helical structures, with a higher occurrence of negative helicity in the northern hemisphere (Seehafer 1990; Pevtsov et al. 1995, 2001; Bao \& Zhang 1998). One possible theoretical explanation of the observed helicity was proposed by Choudhuri (2003), who suggested that the poloidal flux in the solar convection zone (SCZ) gets wrapped around a rising flux tube. Choudhuri et al. (2004) carried out a numerical simulation demonstrating that the observed hemispheric handedness rule can indeed be reproduced by incorporating this mechanism in their dynamo model (Nandy \& Choudhuri 2002; Chatterjee et al. 2004; Choudhuri et al. 2005).

If the magnetic flux in the rising flux tube is nearly frozen, then we expect that the poloidal flux collected by it during its rise through the SCZ would be confined in a narrow sheath at its outer periphery. In order to produce a twist in the flux tube, the poloidal field needs to diffuse from the sheath into the tube by turbulent diffusion. However, turbulent diffusion is strongly suppressed by the magnetic field in the tube. This nonlinear diffusion process was studied in an untwisted flux tube by Petrovay \& Moreno-Insertis (1997) who concluded that a substantial amount of flux may be eroded away from a rising flux tube during the process of its rise through the SCZ. The model was subsequently successfully applied for sunspot decay (Petrovay \& van Driel-Gesztelyi 1997). In the present paper we extend this model by including the poloidal component of the magnetic field (i.e. the field which gets wrapped around the flux tube) and study the evolution of the magnetic field in the rising flux tube, as it keeps collecting more poloidal flux during its rise and as turbulent diffusion keeps acting on it.

The conclusions drawn from our model hinge on some assumptions, especially concerning the subsurface magnetic field structure in the last phases of the rise of the tube. Allowing the various parameters of the model to vary over reasonable ranges, we find that the poloidal field wrapped around the flux tube should be able to penetrate inside the flux tube if the magnetic field falls below the equipartition value in the top layers of the convection zone. On the other hand, if some physical effects keep the magnetic field well above the equipartition value, then the poloidal field may remain confined in a sheath instead 
of penetrating to the core of the flux tube. We expect that much more high-quality magnetogram data will be available in the future and more will be known as to how the current helicity in a sunspot and in an active region varies with radial distance from the centre. This may enable detailed comparisons between theory and observations in future, with the possibility of constraining various parameters in the theory. Our aim at present is only to set up the basic theoretical framework and study some exploratory solutions.

Section 2 presents the mathematical formulation of our problem, while the numerical solutions are presented and discussed in Sect. 3. Section 4 concludes the paper.

\section{Mathematical formulation}

We consider a straight, cylindrical, horizontal magnetic flux tube rising through the solar convective zone. As all variables in this model depend only on the radial distance from the tube axis and on time, we will study the wrapping of the large-scale poloidal field around the flux tube by considering a radially symmetric accretion of azimuthal field by the flux tube. A further complication is the expansion of the flux tube during its rise, due to the decrease of the external pressure. This expansion is assumed to be self-similar. Certainly self-similarity is not a bad assumption. We expect the tube to expand in such a way that the density inside the tube remains homogeneous. A self-similar expansion ensures that.

\subsection{Equations in the comoving Lagrangian frame}

Suppose we formulate our problem in a frame of reference fixed with the centre of the rising flux tube. Substituting a radial expansion velocity

$\boldsymbol{v}=v \boldsymbol{e}_{r}$

and an axisymmetric twisted magnetic field

$\boldsymbol{B}=B_{z} \boldsymbol{e}_{z}+B_{\phi} \boldsymbol{e}_{\phi}$

in the induction equation

$\frac{\partial \boldsymbol{B}}{\partial t}=\nabla \times(\boldsymbol{v} \times \boldsymbol{B})-\nabla \times(\eta \nabla \times \boldsymbol{B})$,

we get the following equations for the axial and poloidal fields in the flux tube:

$\frac{\partial B_{z}}{\partial t}+\frac{1}{r} \frac{\partial}{\partial r}\left(r v B_{z}\right)=\frac{1}{r} \frac{\partial}{\partial r}\left(\eta r \frac{\partial B_{z}}{\partial r}\right)$,

$\frac{\partial B_{\phi}}{\partial t}+\frac{\partial}{\partial r}\left(v B_{\phi}\right)=\frac{\partial}{\partial r}\left[\eta \frac{1}{r} \frac{\partial}{\partial r}\left(r B_{\phi}\right)\right]$.

We first consider what happens in the interior of the flux tube as it expands and is subject to turbulent diffusion. We shall discuss later how accumulation of additional poloidal flux during its rise can be incorporated. The independent variables in the two Eqs. (4) and (5) are $r$ and $t$. Let us assume that the material inside the flux tube expands in a self-similar fashion and use the Lagrangian position coordinate $\xi$ of a fluid element instead of $r$. The initial value of $r$ at time $t=0$ can be taken as the value of $\xi$. Then we can write

$\xi=F(t) r$

where $F(t)$ will have to be a monotonically decreasing function of $t$ for an expanding flux tube. The assumption of selfsimilarity implies that we have to use the same factor $F(t)$ for all fluid elements inside the flux tube to go from the radial coordinate $r$ to the Lagrangian coordinate $\xi$. In Eqs. (4) and (5) we now want to transform from variables $(r, t)$ to $(\xi, t)$. By the chain rule of partial differentiation, it can easily be shown that

$\left(\frac{\partial}{\partial r}\right)_{t}=F\left(\frac{\partial}{\partial \xi}\right)_{t}$

$\left(\frac{\partial}{\partial t}\right)_{r}=\left(\frac{\partial}{\partial t}\right)_{\xi}+\dot{F} r\left(\frac{\partial}{\partial \xi}\right)_{t}$

The velocity is given by

$v=\frac{\mathrm{d} r}{\mathrm{~d} t}=\frac{\mathrm{d}}{\mathrm{d} t}\left(\frac{\xi}{F}\right)=-\frac{\xi}{F^{2}} \dot{F}$.

We also substitute

$B_{z}(r, t)=B_{z}^{\prime}(\xi, t) F^{2}(t)$,

$B_{\phi}(r, t)=B_{\phi}^{\prime}(\xi, t) F(t)$.

On substituting (6)-(11) into (4) and (5), a few steps of straightforward algebra give

$\frac{\partial B_{z}^{\prime}}{\partial t}=F^{2} \frac{1}{\xi} \frac{\partial}{\partial \xi}\left(\eta \xi \frac{\partial B_{z}^{\prime}}{\partial \xi}\right)$,

$\frac{\partial B_{\phi}^{\prime}}{\partial t}=F^{2} \frac{\partial}{\partial \xi}\left[\eta \frac{1}{\xi} \frac{\partial}{\partial \xi}\left(\xi B_{\phi}^{\prime}\right)\right]$.

It is thus clear that $B_{z}^{\prime}(\xi, t)$ and $B_{\phi}^{\prime}(\xi, t)$ do not change with time if $\eta=0$, in accordance with what we expect under the condition of flux freezing.

Equations (12) and (13) clearly should hold from the centre to the outer periphery of the flux tube if we assume it to expand self-similarly.

Now we turn to the problem of how to incorporate in our equations the accretion of poloidal flux to the tube. As the flux tube rises, it collects more poloidal flux, which gets wrapped around it. If we are in the frame of the rising flux tube, it would seem that there is a flow of fluid from the upward direction bringing the poloidal flux. In the downward direction, the surrounding fluid flows away from the flux tube. However, the tension of the poloidal flux makes sure that it gets wrapped around the flux tube, after the reconnection in the wake, as shown in Fig. 4 of Choudhuri (2003). In our one-dimensional model, we can approximately take account of this by assuming that the poloidal flux is brought uniformly from all directions by a radial inward flow with velocity equal to the velocity with which the fluid is flowing from the upward direction. Let us, therefore, consider the nature of flow velocity from the upward direction. Finding the flow past a cylinder is a standard problem in incompressible fluid dynamics (which should hold under the 
subsonic conditions prevailing in our problem) and is discussed in many standard textbooks (see, for example, Choudhuri 1998, Sect. 4.7). To get the velocity in the upward direction, we basically have to substitute $\theta=0$ in the expression (4.54) of Choudhuri (1998) giving the velocity potential and then differentiate it with respect to $r$. This gives

$v=-U_{f t}\left(1-\frac{\xi_{1 / 2}^{2}}{\xi^{2}}\right)$,

where $U_{f t}$ is the velocity of rise of the flux tube and $\xi_{1 / 2}$ is its radius, defined as the place where $B_{z}$ falls to half its maximum value (at the centre of the flux tube). We assume that there is an isotropic radial inward flow given by Eq. (14) towards the flux tube from all directions in the region $\xi>\xi_{1 / 2}$, whereas the flow is zero inside the flux tube $\left(\xi<\xi_{1 / 2}\right)$. Such a flow field certainly has a non-zero divergence and other bad properties. But it should capture the basic physics of poloidal flux advection in a one-dimensional model. We now discuss how we find the advection of the poloidal flux by such a flow field. The term $\partial\left(v B_{\phi}\right) / \partial r$ in Eq. (5) gives the advection of the poloidal field by a radial flow. While deriving Eq. (13) from Eq. (5), we got rid of this term by assuming the self-similar expansion which gives the velocity field (9). If there is an additional velocity field, then the advection term for that velocity would still persist. So we put an additional advection term in (13) in our scaled variable, so that Eq. (13) becomes

$\frac{\partial B_{\phi}^{\prime}}{\partial t}=F^{2} \frac{\partial}{\partial \xi}\left[\eta \frac{1}{\xi} \frac{\partial}{\partial \xi}\left(\xi B_{\phi}^{\prime}\right)\right]-F \frac{\partial}{\partial \xi}\left(v B_{\phi}^{\prime}\right)$.

The factor $F$ in the last term comes from Eq. (7).

\subsection{Input parameters: rise of the flux tube}

To understand the magnetic field evolution in the flux tube, we have to solve Eqs. (12) and (15), starting from some initial configuration and setting suitable boundary conditions at the outer periphery of our region of integration that would allow the free inward advection of the poloidal flux. We also need to specify $F, \eta$ and $v$. Of these, $v$ has already been specified by Eq. (14), although we need to know $U_{f t}$ to get $v$. We now discuss how we obtain $F, U_{f t}$ and $\eta$ in our model. We shall come to a discussion of the initial and boundary conditions later.

As the flux tube rises through the SCZ, we denote its radial distance from the centre of the Sun by $R$. The flux tube begins from the bottom of SCZ at $R=R_{b}$, where its radius is $\xi_{f t}$ and the external density is $\rho_{\mathrm{e}, 0}$. When it rises to $R$ where the external density is $\rho_{\mathrm{e}}$, its radius becomes $r_{f t}$. Since the density inside the flux tube would be very nearly equal to the external density, mass conservation implies

$R_{b} \rho_{\mathrm{e}, 0} \xi_{f t}^{2}=R \rho_{\mathrm{e}} r_{f t}^{2}$.

From (6) it follows that

$F=\frac{\xi_{f t}}{r_{f t}}=\sqrt{\frac{R \rho_{\mathrm{e}}}{R_{b} \rho_{\mathrm{e}, 0}}}$.

Thus, to find $F$ as a function of time, we need to find out how $R$ changes as a function of time and we also require a model of

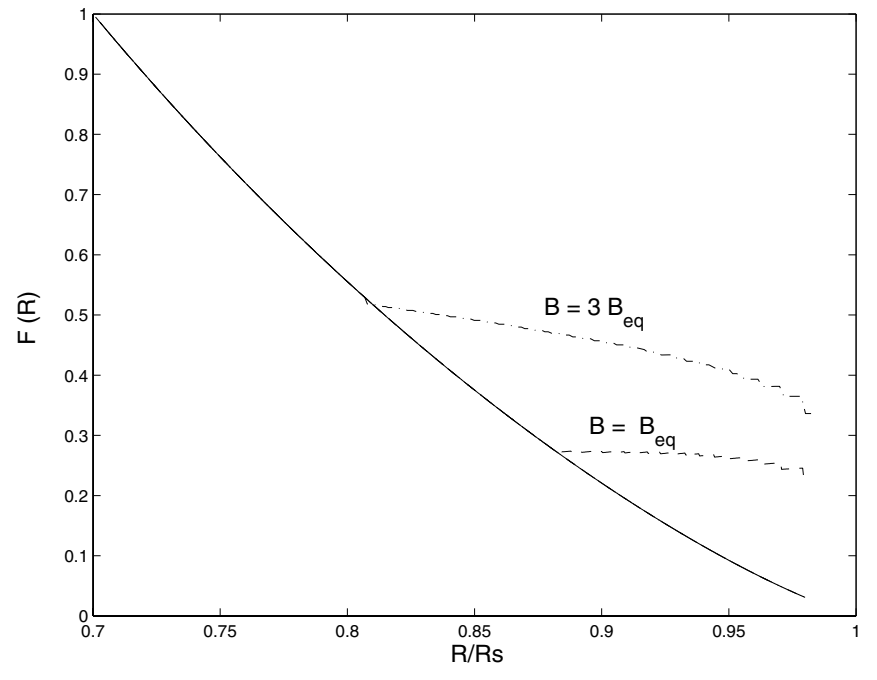

Fig. 1. Variation of the expansion factor $F$ with distance $R$ from the centre of the Sun during the rise of a horizontal flux tube in the convective zone (modelled as an adiabatic envelope). Solid: $F$ calculated from Eq. (16). Dotted and dashed-dotted: $F$ subject to the requirement $B \geq B_{\text {eq }}$ and $B \geq 3 B_{\text {eq }}$, respectively.

the SCZ which will give us the value of $\rho_{\mathrm{e}}$ at that value of $R$. We now discuss how we prescribe a model for SCZ and how we calculate the rise of the flux tube through this SCZ, giving $R$ as a function of time. Since $U_{f t}$ appearing in Eq. (14) is essentially given by $\mathrm{d} R / \mathrm{d} t$, the velocity $v$ as given by Eq. (14) also gets completely specified once we know how $R$ varies with $t$. It is also clear from (16) that $F$ would have a specific value at a certain depth $R$ within the convection zone. The solid line in Fig. 1 shows $F$ as a function of $R$.

How a horizontal magnetic flux tube rises through SCZ can be studied in a fairly straightforward fashion (Moreno-Insertis 1983; Choudhuri \& Gilman 1987). We believe that active regions form by the buoyant rise of a part of a flux tube, while other parts remain anchored at the bottom of SCZ. Studies of the rise of such loops show that the upper parts of the loops move very much like horizontal flux tubes (Moreno-Insertis 1986; Choudhuri 1989; D’Silva \& Choudhuri 1993). We find out how $R$ varies with $t$ by considering the rise of an axisymmetric flux ring. The dynamics of such flux rings has been studied exhaustively by Choudhuri \& Gilman (1987). The forces acting on such a flux ring are (i) magnetic buoyancy, (ii) the Coriolis force, (iii) magnetic tension, and (iv) the drag. The simulations match various aspects of observational data best if the initial magnetic field at the bottom of SCZ is as strong as $10^{5} \mathrm{G}$ (Choudhuri \& Gilman 1987; Choudhuri 1989; D'Silva \& Choudhuri 1993; Fan et al. 1993). For such a strong initial magnetic field, the Coriolis force is unimportant and we neglect it. Also, Choudhuri \& Gilman (1987) made an estimate of magnetic tension compared to magnetic buoyancy. Although magnetic tension may be an appreciable fraction of the magnetic buoyancy at the bottom of SCZ, it becomes negligible in the upper parts of SCZ. So we neglect magnetic tension also. The neglect of the Coriolis force and magnetic tension makes sure that the flux tube moves radially and the problem becomes 
one-dimensional. The only terms in Eq. (4) of Choudhuri \& Gilman (1987) which should be of interest to us are

$2 m_{i} \frac{\mathrm{d}^{2} R}{\mathrm{~d} t^{2}}=-\left(m_{i}-m_{\mathrm{e}}\right) g_{\mathrm{s}}\left(\frac{R_{\odot}}{R}\right)^{2}-\frac{1}{2} C_{D} \rho_{\mathrm{e}} r_{f t}\left(\frac{\mathrm{d} R}{\mathrm{~d} t}\right)^{2}$,

where $g_{\mathrm{s}}$ is the gravity at the solar surface, whereas $m_{i}=\pi r_{f t}^{2} \rho_{i}$ and $m_{\mathrm{e}}=\pi r_{f t}^{2} \rho_{\mathrm{e}}$ are respectively mass per unit length of the flux tube and the displaced fluid. The dimensionless drag coefficient $C_{\mathrm{D}}$ is found to have a value of around 0.4 in laboratory experiments (Goldstein 1938; Schlichting 1979). Dividing Eq. (17) by $2 \pi r_{f t}^{2} \rho_{\mathrm{e}}$, we get

$\frac{\mathrm{d}^{2} R}{\mathrm{~d} t^{2}}=\frac{\rho_{\mathrm{e}}-\rho_{i}}{2 \rho_{\mathrm{e}}} g_{\mathrm{s}}\left(\frac{R_{\odot}}{R}\right)^{2}-\frac{C_{\mathrm{D}}}{4 \pi r_{f t}}\left(\frac{\mathrm{d} R}{\mathrm{~d} t}\right)^{2}$.

It may be noted that $\rho_{i} \approx \rho_{\mathrm{e}}$ so that we treat them differently only when we have to consider the difference between them.

The magnetic buoyancy factor $\left(\rho_{\mathrm{e}}-\rho_{i}\right) /\left(2 \rho_{\mathrm{e}}\right)$ depends on the background model of SCZ that we use. If we assume the temperature gradient to be exactly equal to the adiabatic gradient, then we end up with a polytropic model for SCZ. Choudhuri \& Gilman (1987) used a polytropic model with a choice of parameters which gave a close fit to more detailed models of SCZ. The model is described through Eqs. (10)-(12) of Choudhuri \& Gilman (1987), with the values of parameters listed at the beginning of Sect. III. We use this model of SCZ here. We also need to use some suitable thermal condition to calculate the magnetic buoyancy factor $\left(\rho_{\mathrm{e}}-\rho_{i}\right) /\left(2 \rho_{\mathrm{e}}\right)$. Choudhuri \& Gilman (1987) presented results for three thermal conditions. We present our calculations here for the simplest case of the flux tube being in thermal equilibrium with the surrounding, although we have done some calculations for the other thermal conditions and found the results to be qualitatively similar. For the case of thermal equilibrium, the magnetic buoyancy factor is given by

$\frac{\rho_{\mathrm{e}}-\rho_{i}}{2 \rho_{\mathrm{e}}}=\frac{B_{0}^{2}}{16 \pi p_{\mathrm{e}, 0}}\left(\frac{T}{T_{0}}\right)^{(2-\gamma) /(\gamma-1)}\left(\frac{R}{R_{b}}\right)^{2}$.

Knowing the temperature $T$ at the position $R$ from the SCZ model, we can use Eq. (19) to calculate the magnetic buoyancy factor of a flux tube at $R$, which started with initial magnetic field $B_{0}$. Calculating magnetic buoyancy in this way and using Eq. (16) to find $r_{f t}$ at the position $R$, we can integrate Eq. (18) to find how $R$ changes with time. From the variation of $R$ with time, on making use of Eq. (16), we can find how $F(t)$ varies with time (Fig. 1). The velocity $U_{f t}$ in Eq. (14) is essentially the rise velocity of the flux tube, i.e.

$U_{f t}=\frac{\mathrm{d} R}{\mathrm{~d} t}$

So, once we know how $R$ changes with $t$, we can use Eqs. (14) and (20) to obtain $v$ that appears in Eq. (15).

\subsection{Input parameters: turbulent diffusivity}

As we already pointed out, we need to keep solving Eqs. (12) and (15), as the flux tube rises, to find out how the magnetic field evolves. Now that we know how to find $F(t)$ and $v$ at any time step, we only need to specify the turbulent diffusivity $\eta$. For this we follow the approach of Petrovay \& Moreno-Insertis (1997) and take $\eta$ to be given by the expression

$\eta=\frac{\eta_{0}}{1+\left|B / B_{\mathrm{eq}}\right|^{\chi}}$

where $B=\sqrt{B_{z}^{2}+B_{\phi}^{2}}$ is the amplitude of the magnetic field and $B_{\text {eq }}$ is the equipartition magnetic field. We use the convection zone model of Unno et al. (1985) to obtain $B_{\text {eq }}$ at different positions $R$ within SCZ. Most of our calculations are done by taking $x=2$ (except in the case presented in Fig. 3 ). We specify $\eta_{0}$ exactly the same way as Petrovay \& Moreno-Insertis (1997). If $H$ is the pressure scale height, then we take

$\eta_{0}=\eta_{00}\left(\frac{r_{f t}}{H}\right)^{4 / 3}$

with

$\eta_{00}=3 \times 10^{12} \mathrm{~cm}^{2} \mathrm{~s}^{-1}$.

Chatterjee et al. (2004) found that solar dynamo models give the best fits with observations for a value of diffusion of this order. It may be noted that we use Eq. (22) only when $r_{f t}<H$, which is the case for typical flux tubes rising through the bulk of SCZ except the uppermost layers. There we take $\eta_{0}=\eta_{00}$. However, even the validity of Eq. (18) becomes questionable if $r_{f t}>H$.

Now Eqs. (12) and (15) are a set of nonlinear fluxconserving equations that may be solved by an explicit two-step Lax-Wendroff scheme. The time step $\Delta t$ obeys the stability condition $\Delta t<\min \left[(\Delta \xi)^{2} / \eta_{0}, \Delta \xi / U_{f t}\right]$, where $U_{f t}$ is the velocity of rise calculated from Eq. (18) and $\eta_{0}$ is given by Eq. (22). We use a non-uniform but steady spatial grid with 1500 points which has a finer resolution of $\Delta \xi \sim 2.4 \mathrm{~km}$ for $\xi<3000 \mathrm{~km}$ and increasing successively by $2 \%$ thereafter up to $\xi=20000 \mathrm{~km}$.

\subsection{Initial and boundary conditions}

Now that we have described how all the various terms appearing in Eqs. (12) and (15) are specified or can be obtained, we only have to discuss the initial and boundary conditions used to solve these equations. Suppose we want to consider the buoyant rise of a flux tube carrying initial flux $\Psi$ with an initial magnetic field $B_{0}$ at the bottom of SCZ. The initial radius of the flux tube is obviously $\xi_{f t}=\sqrt{\Psi / \pi B_{0}}$. We take the initial condition that $B_{z}=B_{0}$ inside $r<\xi_{f t}$ and $B_{z}=0$ outside, whereas $B_{\phi}$ is initially taken to be zero everywhere. The integration region over which Eqs. (12) and (15) are integrated extends to $r_{\text {out }}$ which is typically taken at $10 \xi_{f t}$. The solutions are not very sensitive as to what boundary conditions we use for $B_{z}^{\prime}$. However, since the flux tube keeps on acquiring poloidal flux which must be advected inward through the boundary of the integration region, the boundary condition on $B_{\phi}^{\prime}$ is quite important. We assume that the SCZ is filled with a uniform horizontal magnetic field of $1 \mathrm{G}$, which gets wrapped around the rising flux tube. In order to achieve this, we have to continuously advect $B_{\phi}=1 \mathrm{G}$ 

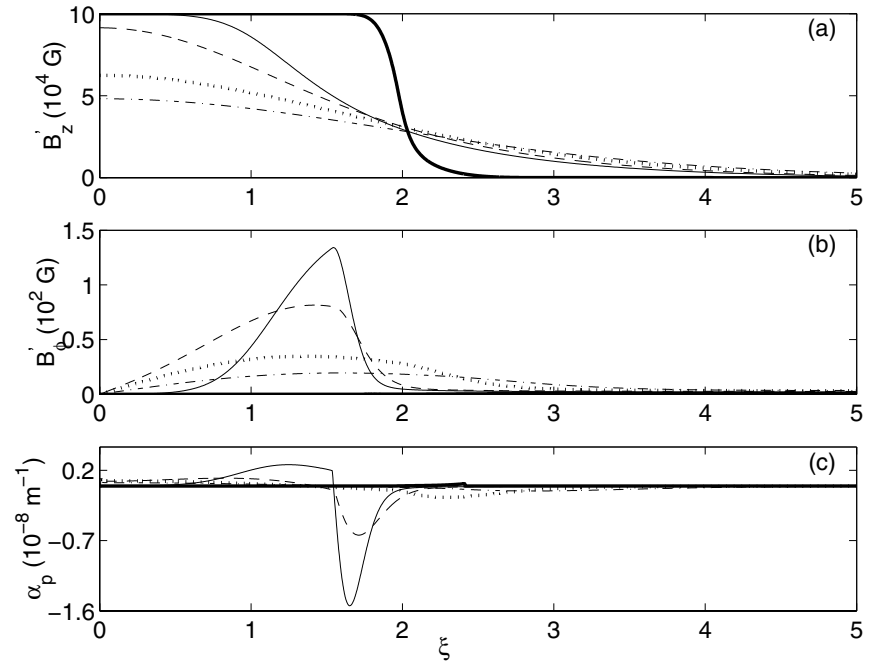

Fig. 2. Plots of $B_{z}^{\prime}, B_{\phi}^{\prime}$ and $\alpha_{p}$ as functions of $\xi$ for a rising flux tube with $x=2$ (case A). The different curves correspond to the profiles of these quantities at the following positions of the flux tube: $0.7 R_{\odot}$ (thick solid), $0.85 R_{\odot}$ (solid), $0.9 R_{\odot}$ (dashed), $0.95 R_{\odot}$ (dotted), $0.98 R_{\odot}$ (dash-dotted). The flux tube reaches these positions at times 0 days, 5.3 days, 6.6 days, 7.9 days and 8.7 days after the initial start. The values of $\left(B / B_{\text {eq }}\right)$ at the centres of these flux tubes at these positions are 10,1.72, 0.64, 0.098, 0.015 .

through the outer boundary of the integration region. Looking at Eq. (11), we realize that the appropriate boundary condition at $r=r_{\text {out }}$ is going to be

$B_{\phi}^{\prime}=1 / F(t) \mathrm{G}$.

\section{Results and discussion}

Flux tube simulations (Choudhuri \& Gilman 1987; Choudhuri 1989; D’Silva \& Choudhuri 1993; Fan et al. 1993) have suggested that theory matches various aspects of observations best if the flux tubes start with initial fields of order $10^{5} \mathrm{G}$ at the bottom of SCZ. We, therefore, carry out all our calculations by taking an initial magnetic field of $10^{5} \mathrm{G}$. The typical flux carried by a large sunspot is $10^{22} \mathrm{Mx}$. So we present results for a flux tube with such flux, which implies that the initial radius at the bottom of SCZ is $R_{b}=1.78 \times 10^{3} \mathrm{~km}$. We study the rise of the flux tube by numerically integrating Eq. (18) with the magnetic buoyancy given by Eq. (19). While the flux tube rises, we study the evolution of the magnetic field in the flux tube by solving Eqs. (12) and (15) using a Lax-Wendroff scheme, with $F(t)$ given by Eq. (16), $v$ by Eq. (14) and $\eta$ by Eq. (21).

Figures 2 to 6 show the magnetic fields of the rising flux tube at depths $R=0.7 R_{\odot}, 0.85 R_{\odot}, 0.9 R_{\odot}, 0.95 R_{\odot}, 0.98 R_{\odot}$. Profiles of $B_{z}^{\prime}$ as functions of $\xi$ are shown in the top panels, whereas the middle panels show the profiles of $B_{\phi}^{\prime}$. As these panels show the rescaled variables, the actual field strengths can be calculated from them using Fig. 1 to read off $F$ for each curve, and plugging that value into Eqs. (10) and (11). Note that $B_{\phi}^{\prime}$ tends to the value given by (24) for large $\xi$. Since this is much smaller than what $B_{\phi}^{\prime}$ becomes near the flux tube
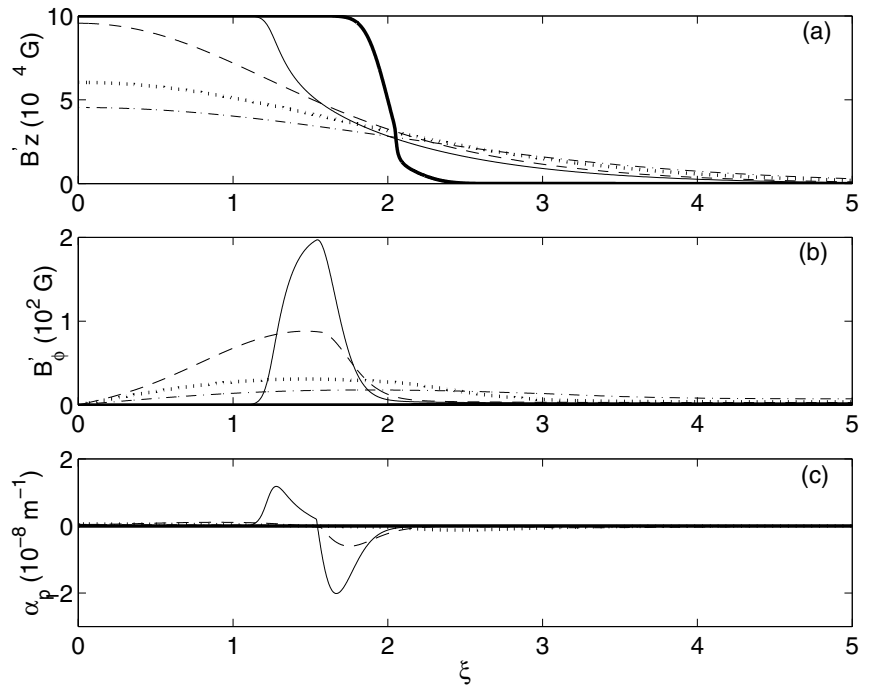

Fig. 3. Same as Fig. 2 but with $\varkappa=5$. The flux tube reaches positions $0.0 .7 R_{\odot}, 0.85 R_{\odot}, 0.9 R_{\odot}, 0.95 R_{\odot}, 0.98 R_{\odot}$ at times 0 days, 5.2 days, 6.6 days, 7.9 days and 8.7 days after the initial start. The values of $\left(B / B_{\text {eq }}\right)$ at the centres of these flux tubes at these positions are $10,1.74,0.66,0.095$ and 0.018 respectively.
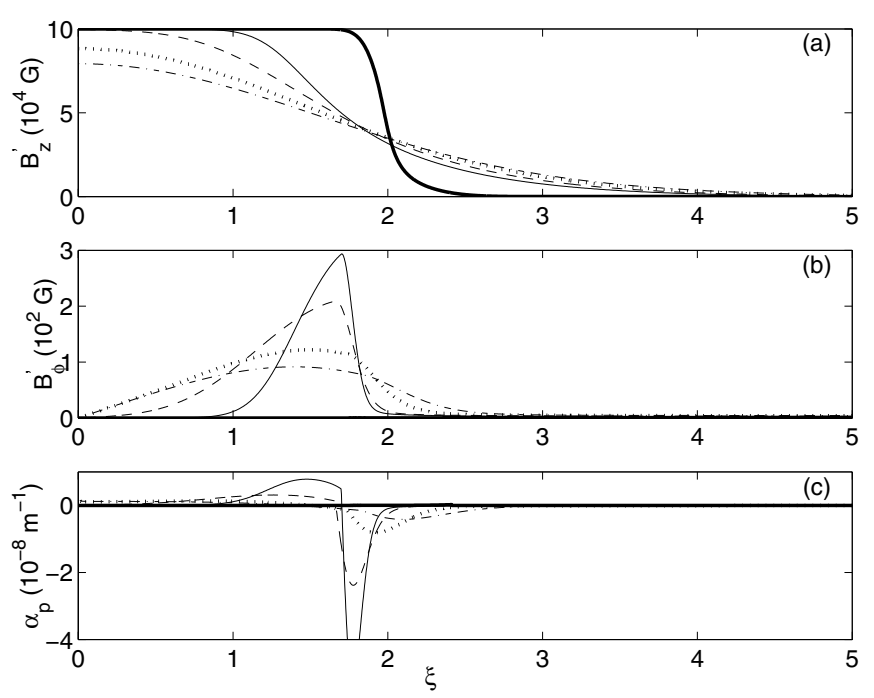

Fig. 4. Same as Fig. 2 except $\eta_{00}=10^{12} \mathrm{~cm}^{2} / \mathrm{s}$. The flux tube reaches positions $0.7 R_{\odot}, 0.85 R_{\odot}, 0.9 R_{\odot}, 0.95 R_{\odot}, 0.98 R_{\odot}$ at times 0 days, 5.2 days, 6.6 days, 7.9 days and 8.7 days after the initial start. The values of $\left(B / B_{\text {eq }}\right)$ at the centres of these flux tubes at these positions are $10,1.72,0.69,0.14$ and 0.03 respectively.

boundary, it appears in Figs. 2 to 6 as if $B_{\phi}^{\prime}$ is going to zero for large $\xi$, although this is not the case.

The bottom panels provide plots of

$\alpha_{p}=(\nabla \times \boldsymbol{B})_{z} / B_{z}$

since this is the quantity that essentially all photospheric measurements of the current helicity actually determine (Leka \& Skumanich 1999; Burnette et al. 2004). It is easy to see that $\alpha_{p}$, which has the dimension of $1 /$ length, is invariant to the rescaling of the radial coordinate in our flux tube. 

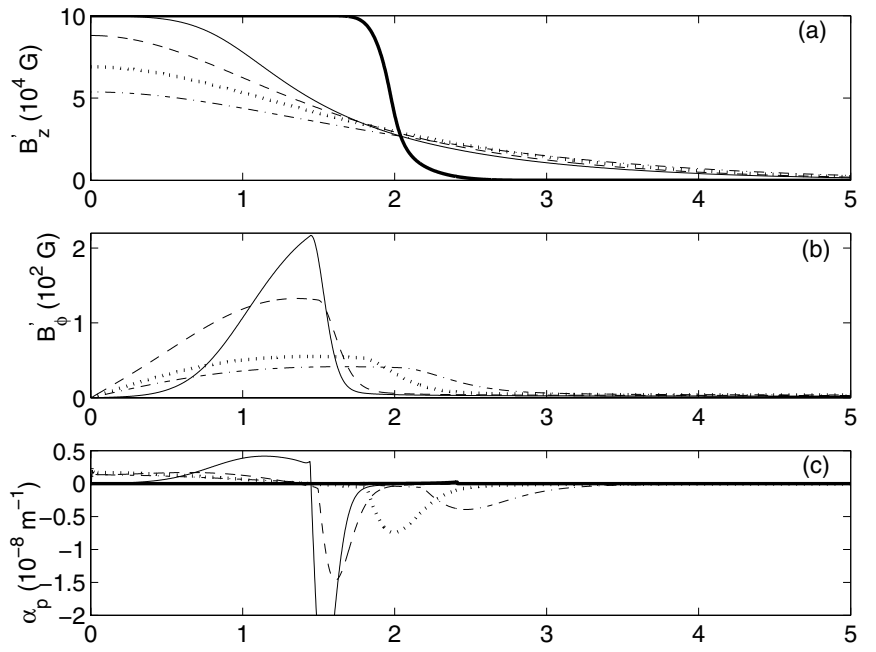

Fig. 5. Plots of $B_{z}^{\prime}, B_{\phi}^{\prime}$ and $\alpha_{p}$ as functions of $\xi$ for a rising flux tube. The field inside the tube is not allowed to decrease below $B_{\text {eq }}$ (case B1). The different curves correspond to the profiles of these quantities at the following positions of the flux tube: $0.7 R_{\odot}$ (thick solid), $0.85 R_{\odot}$ (solid), $0.9 R_{\odot}$ (dashed), $0.95 R_{\odot}$ (dotted), $0.98 R_{\odot}$ (dash-dotted). The flux tube reaches these positions at times 0 days, 5.2 days, 6.6 days, 7.7 days and 8.2 days after the initial start. The values of $\left(B / B_{\text {eq }}\right)$ at the centres of these flux tubes at these positions are $10,1.72,1.0,1.0,1.0$ respectively.
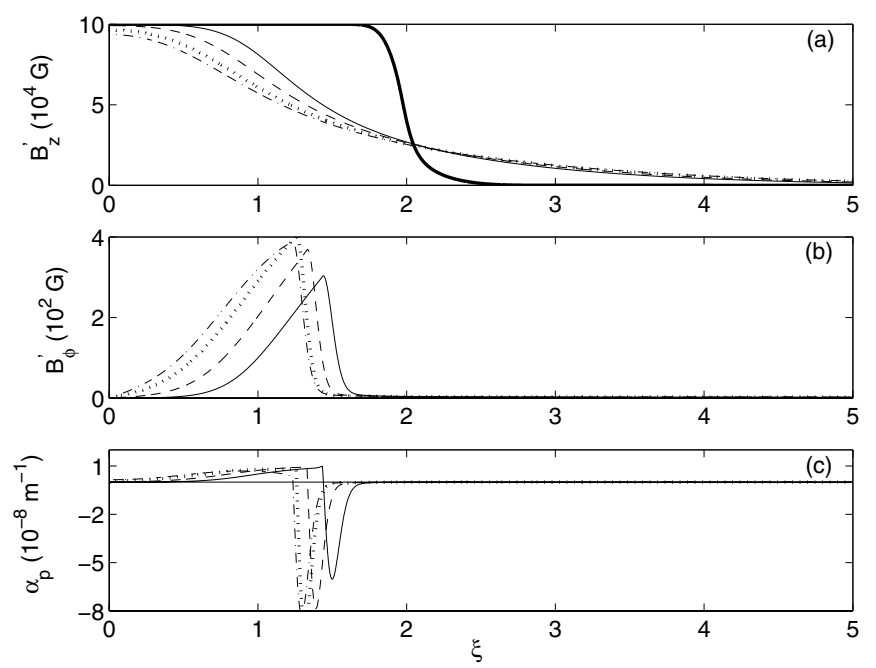

Fig. 6. Plots of $B_{z}^{\prime}, B_{\phi}^{\prime}$ and $\alpha_{p}$ as functions of $\xi$ for a rising flux tube. The field inside the flux tube is not allowed to decrease below $3 B_{\text {eq }}$ at any height (case B3). The different curves correspond to the profiles of these quantities at the following positions of the flux tube: $0.7 R_{\odot}$ (thick solid), $0.85 R_{\odot}$ (solid), $0.9 R_{\odot}$ (dashed), $0.95 R_{\odot}$ (dotted), $0.98 R_{\odot}$ (dash-dotted). The flux tube reaches these positions at times 0 days, 5.2 days, 6.2 days, 6.8 days and 7.0 days after the initial start. The values of $\left(B / B_{\mathrm{eq}}\right)$ at the centres of these flux tubes at these positions are $10,3,3,3,3$ respectively.

\subsection{Case A: diffuse magnetic field near the surface}

It should be evident from Fig. 2 that diffusion does not play a significant role until the flux tube reaches $0.85 R_{\odot}$. The diffusion in the deeper layers turns out to be negligible due to two factors: (i) since $\left(B / B_{\text {eq }}\right)$ is large, the quenching included in Eq. (21) is quite efficient; (ii) since $\left(r_{f t} / H\right)$ is small, $\eta_{0}$ as given by Eq. (22) turns out to be small. As a result of the low diffusion, the toroidal field $B_{z}^{\prime}$ does not change much, whereas the poloidal flux $B_{\phi}^{\prime}$ remains confined in a sheath at the outer periphery of the flux tube (since the low diffusion does not allow $B_{\phi}^{\prime}$ to diffuse inward). After the flux tube has risen above $0.85 R_{\odot}$, the toroidal magnetic field inside the flux tube becomes comparable to the equipartition field $B_{\text {eq. Then }}$ diffusion is able to affect the magnetic field much more, since $\eta$ given by Eq. (21) becomes much larger due to the diminishing role of magnetic suppression of diffusion. As a result, $B_{z}^{\prime}$ starts diffusing while the flux tube rises through the top layers of $\mathrm{SCZ}$, whereas $B_{\phi}^{\prime}$ penetrates to the core of the flux tube instead of remaining confined to a sheath at the other periphery.

In the very top layers, however, the cross-section of the flux tube becomes enormous in our model, leading to small values of $F(t)$ and again making diffusion less important, as can be seen from Eqs. (12) and (15). This is why we find that the magnetic field profile has not evolved that much from $0.95 R_{\odot}$ to $0.98 R_{\odot}$. It may be noted that the profile of $B_{z}^{\prime}$ no longer has a sharp edge after the flux tube rises above $0.9 R_{\odot}$, but spreads around. In the realistic situation, however, we expect the evolution of $B_{z}^{\prime}$ in the immediate vicinity of the flux tube to be much more complicated. In the frame of the flux tube, it would appear that the surrounding fluid is flowing inward from the upward direction and this would prevent the spread of $B_{z}^{\prime}$ in the upward direction. On the other hand, the surrounding fluid moves away from the flux tube in the downward direction and would carry away $B_{z}^{\prime}$ with it, leading to a larger spread of $B_{z}^{\prime}$ in the downward direction. In a one-dimensional model, the best way of capturing the average behaviour of $B_{z}^{\prime}$ may be not to include any velocity field in the surrounding fluid, as we have done by not including any advection term in Eq. (12). However, an advection term is included in the evolution Eq. (15) for $B_{\phi}^{\prime}$. Because of the topology of magnetic field lines, the poloidal field cannot be advected freely in the downward direction, as can be seen in Fig. 4 of Choudhuri (2003). After a reconnection in the wake, a poloidal field line should remain wrapped around the flux tube due to its tension. Hence the behaviour of the poloidal field is best captured in a one-dimensional model by including a uniform inward flow from all the directions. To treat the evolution of the magnetic field more realistically, it would be necessary to go beyond one-dimensional models. Some of us are now involved in developing a two-dimensional model of this problem. However, the one-dimensional model should capture some of the basic effects correct to the right order of magnitude.

\subsection{Parameter dependence}

Since many parameters of the problem are not known well, one very important question is whether the results presented in Fig. 2 are sufficiently generic or would change substantially on changing the various parameters. Figure 2 presents results obtained by assuming thermal equilibrium of the flux tube with the surroundings. Choudhuri \& Gilman (1987) presented results for two other thermal conditions: by assuming the interior of the flux tube as adiabatic with and without a superadiabatic 
gradient in the surrounding SCZ. We carried out some calculations with these two thermal conditions as well. There are virtually no changes till the flux tube rises beyond $0.9 R_{\odot}$. After that, the flux tube rises faster in these two cases compared to the case of thermal equilibrium and diffusion has less time to act. Therefore, we find the effect of diffusion a little bit less when the flux tube reaches the topmost layers of SCZ. Figure 3 presents results obtained by using a quenching index $\varkappa=5$ instead of $x=2$ used in Fig. 3, whereas all the other things remain the same as in Fig. 2. Again the results are not qualitatively different. We also study what happens if the diffusivity is made smaller compared to what is prescribed in (23). Figure 4 presents results obtained with $\eta_{00}=10^{12} \mathrm{~cm}^{2} \mathrm{~s}^{-1}$, whereas all the other things remain the same as in Fig. 2. As expected, we find the diffusion somewhat less. Otherwise, results are not qualitatively different. We thus conclude that the results presented in Fig. 2 are sufficiently generic for a reasonable range of parameters, as long as we hold on to our basic model of the horizontal flux tube rise.

\subsection{Case B: concentrated magnetic field near the surface}

One of the unsatisfactory aspects of the horizontal flux tube rise model is that the magnetic field falls to very small values in the top layers of SCZ when the flux tube expands enormously by moving to a low-density region. In the case presented in Fig. 2, our model predicts that the magnetic field inside the flux tube when it reaches $0.98 R_{\odot}$ is about $400 \mathrm{G}-$ an order of magnitude smaller than a typical sunspot field. Since such a field is much weaker than the equipartition field, it should be clear from Eq. (21) that the magnetic quenching of diffusion becomes completely negligible and diffusion is able to act without being inhibited by the presence of the magnetic field. The presence of $3000 \mathrm{G}$ magnetic fields in sunspots is a compelling proof that magnetic fields may never fall to such low values; in fact, at least in photospheric layers, they remain well above the equipartition value (the magnetic field inside a typical sunspot being about thrice the equipartition field). The nonaxisymmetric flux tube simulations (Choudhuri 1989; D'Silva \& Choudhuri 1993; Fan et al. 1993) show that the fluid drains from the tops of rising magnetic loops making the magnetic field there stronger. Additionally, effects like convective collapse (Steiner 2003) can be operative near the solar surface to enhance the magnetic field. Longcope \& Choudhuri (2002) have argued that flux tubes get distorted by convective turbulence in the top layers of SCZ, leading to the observed scatter of tilt angles around what would be expected from Joy's law. Such buffeting of flux tubes by turbulence can also cause an enhancement of magnetic field by stretching.

Most of the flux rise simulations are based on the thin flux tube approximation, which should be valid during the rise of flux tubes to about $0.9 R_{\odot}$ during which the magnetic field remains sufficiently strong to stay relatively unaffected by the surrounding turbulence. So we can presumably trust the flux rise simulations through the deeper layers of convection. However, our understanding of what happens during the rise of flux tubes through the top layers of SCZ is extremely poor. Techniques of local helioseismology, such as time-distance seismology, have now evolved to a stage when the direct study of subsurface structures in emerging active regions has become possible (Kosovichev et al. 2000; Hughes et al. 2005). There is thus hope that this issue may be resolved in the not too distant future. In any case, the observation of sunspot magnetic fields indicates that there may be effects which prevent the magnetic field from falling to values lower than the equipartition field even in the top layers of SCZ.

We now present some calculations by artificially not allowing the magnetic field to fall below the equipartition value. Suppose the magnetic field in the interior of the flux tube falls to a value $s B_{\text {eq }}$ at some depth ( $s$ being a numerical factor of the order of unity). We assume that the magnetic field inside the flux tube remains $s B_{\text {eq }}$ in the higher layers as it rises further. Here $B_{\text {eq }}$ is the local equipartition value at the particular depth. If this is the case, then magnetic buoyancy would be given by

$\frac{\rho_{\mathrm{e}}-\rho_{i}}{2 \rho_{\mathrm{e}}}=\frac{s^{2} B_{\mathrm{eq}}^{2}}{16 \pi p_{\mathrm{e}}}$

instead of Eq. (19). While we calculate the rise of the flux tube by using this expression of magnetic buoyancy, we cannot allow the cross-section to expand indefinitely if the magnetic field has to remain $s B_{\text {eq }}$. Instead of Eq. (16), we calculate $F(t)$ by using the relation

$F(t)=\sqrt{\frac{s B_{\mathrm{eq}}}{B_{0}}}$

(dashed and dash-dotted lines in Fig. 1). By calculating $F(t)$ in this way, we solve Eqs. (12) and (15) to find the evolution of the magnetic field. It may be noted that in the expression of drag in Eq. (18) also, we have to use $r_{f t}=\xi_{f t} / F(t)$ with $F(t)$ given by Eq. (27).

Results for $s=1$ (case B1) and $s=3$ (case B3) are shown in Figs. 5 and 6 respectively. As in Figs. 2-4, we plot $B_{z}^{\prime}, B_{\phi}^{\prime}$ and $\alpha_{p}$ as functions of $\xi$ at depths $0.7 R_{\odot}, 0.85 R_{\odot}, 0.9 R_{\odot}$, $0.95 R_{\odot}$ and $0.98 R_{\odot}$. The times taken to reach these depths are given in the figure captions. Comparing with the times given in the caption of Fig. 2, it will be seen that the flux tubes have risen faster through the top layers of SCZ, since magnetic buoyancy has remained stronger. The diffusion remains significantly quenched if the magnetic field stays higher than $B_{\text {eq }}$ and has also less time to act because the flux tube rises faster. As a result, we see that the effect of diffusion is somewhat less for case B1 and drastically less for case B3 compared to case A. We see in Fig. 6 that $B_{z}^{\prime}$ has not diffused much and $B_{\phi}^{\prime}$ has remained confined in a narrow sheath at the boundary of the flux tube, being unable to diffuse inward.

\subsection{Current helicity}

Observations of the current helicity parameter $\alpha_{p}$ indicate that its typical average value in an active region is on the order of $10^{-8} \mathrm{~m}^{-1}$ (Pevtsov et al. 1995; van Driel-Gesztelyi et al. 2003; Burnette et al. 2004). Inspecting the lower panels of Figs. 2 to 6 one finds that the typical value of $\alpha_{p}$ in the internal parts of the 
flux tube is of order $\sim 10^{-8} \mathrm{~m}^{-1}$ at a depth of $0.85 R_{\odot}$ in all the cases studied. However, as the flux reaches the solar surface, in all the cases except the case B3 presented in Fig. 6, the $B_{\phi}$ component spreads out due to diffusion and its gradient becomes smaller, reducing $\alpha_{p}$ by about one order of magnitude. Only if the magnetic field inside the flux tube remains stronger than the equipartition field (the case B3 represented in Fig. 6), the $B_{\phi}$ component is unable to diffuse inside so that its gradient remains strong and $\alpha_{p}$ is of order $\sim 10^{-8} \mathrm{~m}^{-1}$ even near the surface. This suggests that our case B3 may be closest to reality, i.e. during the rise of the flux tube from $0.9 R_{\odot}$ to $0.98 R_{\odot}$ effective flux concentration processes are at work, keeping the field strength at a value somewhat above the equipartition level. This is consistent with the notion that the main flux concentration effect at work here is turbulent concentration (i.e. flux expulsion by the turbulent eddies: Proctor \& Weiss 1982), while convective collapse and thermal relaxation (Steiner 2003) are restricted to the shallowest layers above $0.98 R_{\odot}$.

Note, however, that in the calculations presented here the amplitude and sign of the poloidal field was assumed not to depend on depth. For alternative assumptions, significantly different current helicities may result, so the above conclusion should be treated with proper reservation. Details of the radial dependence of the poloidal field strength may strongly depend on the dynamo model.

A more robust feature of the current helicity distributions, present in all the lower panels of our plots, is the presence of a ring around the tube with a current helicity of the opposite sense. This is clearly the consequence of the fact that on the outer side of the accreted sheath the radial gradient of the azimuthal field, and thus the axial current, is negative. This is an inevitable corollary of the present mechanism of producing twist in active regions. A rather strong prediction of this model is, therefore, that a ring of reverse current helicity should be observed on the periphery of active regions, somewhere near the edge of the plage.

\subsection{Flux loss from the rising tube}

Petrovay \& Moreno-Insertis (1997) came to the conclusion that a considerable amount of magnetic flux is lost during the rise of a flux tube through the SCZ (see their Fig. 10). This conclusion was based on an approximate expression of inward velocity of turbulent erosion and involved various uncertainties. One important question is whether our more careful calculations show similar flux losses. To make an estimate of the flux loss, we keep calculating the flux

$\psi_{0}=2 \pi \int_{0}^{\xi_{0}} B_{z}^{\prime}(\xi) \xi \mathrm{d} \xi$

within the initial Lagrangian radius $\xi_{0}$ of the flux tube. Figure 7 gives plots as $\psi_{0}$ as a function of the position in the SCZ for the four cases presented in Figs. 2, 4-6. We find that the flux loss is much less if the magnetic field is not allowed to fall below $B_{\text {eq. }}$. Even in the case when the magnetic field is allowed to fall to rather low values (the solid line in Fig. 7), the flux loss is nowhere as substantial as inferred by Petrovay \& Moreno-Insertis (1997). The main reason for this discrepancy

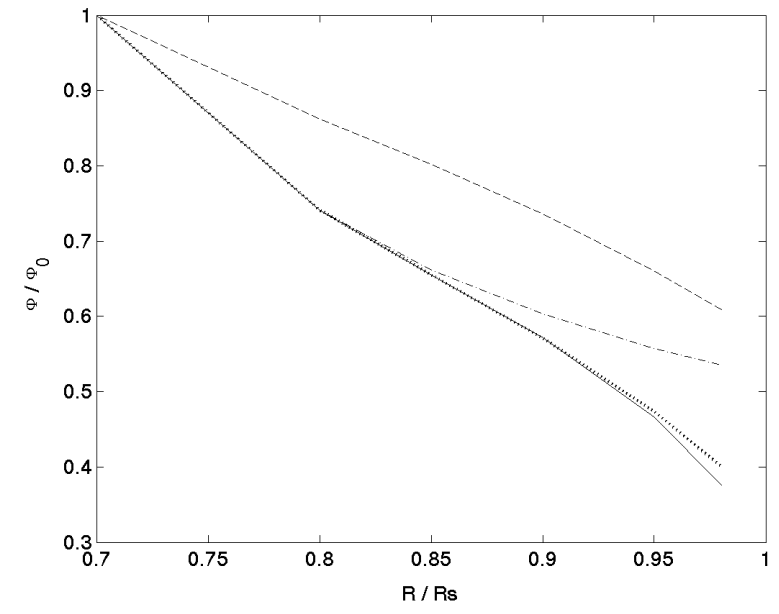

Fig. 7. Flux loss as a function of height $R$ inside the convection zone for the four cases presented in Figs. 2 (solid), 4 (dashed), 5 (dotted), 6 (dash-dotted).

is that we do not make use of the assumption of a continuous "re-initialization" of the decay, as it was done in that paper. The basis of that assumption was that the flow of external fluid relative to the tube would instantly remove all magnetic flux lost from the tube. However, in the presence of an azimuthal field component, field line topology is expected to inhibit such flux removal. We thus conclude that flux loss during the rise of a flux tube is much less significant than what Petrovay \& Moreno-Insertis (1997) found in their calculations. This provides a justification of the flux rise calculations based on the thin flux tube equation, where it is assumed that the magnetic field is frozen during the rise of the flux tube. If the above calculations are repeated for the flux tube rising adiabatically through the convection zone, then the flux losses are somewhat less. For example, in the case B3, the flux loss is then $41.2 \%$ instead of $46.5 \%$ during the isothermal rise.

\section{Conclusion}

Alternative mechanisms for the origin of twist in active region magnetic fields include buffeting of the rising flux tubes by helical turbulent motions (Longcope et al. 1998); the effect of Coriolis force on flows in rising flux loops (Fan \& Gong 2000); differential rotation (DeVore 2000); and helicity generation by the solar dynamo (Seehafer et al. 2003). The relative importance of these processes is currently a subject of debate (Holder et al. 2004). It is likely that the accretion of poloidal fields during the rise of a flux tube is just one contribution to the development of twist. Its importance may also be reduced by 3D effects: considering the rise of a finite flux loop instead of an infinite horizontal tube, the possibility exists for the poloidal field to "open up", giving way to the rising loop with less flux being wrapped around it. It is left for later multidimensional analyses of this problem to determine the importance of any such reduction. In any case, the results presented above indicate that the contribution of poloidal field accretion to the development of twist can be quite significant, and under favourable circumstances it can potentially account for most of the current helicity observed in active regions. 
In our calculations we found that while the flux tube rises to a depth of about $0.85 R_{\odot}$, the effect of diffusion is small and the poloidal field remains confined in a narrow sheath at the periphery of the flux tube. Afterwards, if the magnetic field is allowed to fall to very low values as suggested by simple flux tube rise simulations (case A), then the effect of diffusion is considerable and the poloidal field is able to penetrate into the interior of the flux tube. On the other hand, if various effects in the top layers of SCZ keep the magnetic field above $B_{\text {eq }}$ (case B1) and $3 B_{\text {eq }}$ (case $\mathrm{B} 3$ ), then diffusion is less effective; in case B3 the poloidal field remains confined in the sheath. For a poloidal field strength independent of depth, as assumed in these calculations, the best agreement with the observed current helicity values is found for case B3.

One rather strong prediction of our model is the existence of a ring of reverse current helicity on the periphery of active regions. On the other hand, the amplitude of the resulting twist (as measured by the mean current helicity in the inner parts of the active region) depends sensitively on the assumed structure (diffuse vs. concentrated/intermittent) of the active region magnetic field right before its emergence, and on the assumed vertical profile of the poloidal field. Nevertheless, a mean twist comparable to the observations can result rather naturally in the model with perhaps the most plausible choice of assumptions (case B3).

Thus, by studying the distribution of the azimuthal magnetic field in sunspots and active regions, and by simultaneously studying the subsurface magnetic field structure in emerging active regions by means of local helioseismology, it may be possible to test the present model and to throw some light on the conditions prevailing during the last phase of flux tube rise through the top layers of SCZ. We hope that magnetogram data will improve significantly in the next few years and it will be possible to draw meaningful inferences. Since at present we have very poor understanding of the nature of the magnetic field or the effect of turbulence when a flux tube rises through layers immediately below the solar surface, indirect inferences from such observations of sunspots are of great importance.

Lastly, we have used a simplifying assumption that the poloidal field that gets wrapped around the flux tube has a constant value of $1 \mathrm{G}$ inside the SCZ. Dynamo models like the model of Choudhuri et al. (2005) can be used to calculate the distribution of the poloidal field inside the SCZ during different phases of the solar cycle. More detailed calculations of helicity can be done by using such poloidal field distributions instead of using the simple boundary condition (24). Since some groups have started reporting on the possible cycle variation of helicity on the basis of the observational data (Bao et al. 2000; Hagino \& Sakurai 2005), such calculations may become relevant in future when more detailed observational data are available.

Acknowledgements. We thank Dibyendu Nandy and an anonymous referee for valuable comments. This research was carried out in the framework of the Indo-Hungarian Inter-Governmental Science \& Technology Cooperation scheme, with the support of the Hungarian
Research \& Technology Innovation Fund and the Department of Science and Technology of India. K.P. acknowledges support from the UK Particle Physics and Astronomy Research Council under grant no. PP/X501812/1, from the ESMN network supported by the European Commission, and from the OTKA under grant no. T043741. P.C. acknowledges financial support from Council for Scientific and Industrial Research, India under grant no. 9/SPM-20/2005-EMR-I.

\section{References}

Bao, S., \& Zhang, H. 1998, ApJ, 496, L43

Bao, S. D., Ai, G. X., \& Zhang, H. Q. 2000, J. Ap. Astron., 21, 303

Burnette, A. B., Canfield, R. C., \& Pevtsov, A. A. 2004, ApJ, 606, 565

Chatterjee, P., Nandy, D., \& Choudhuri, A. R. 2004, A\&A, 427, 1019

Choudhuri, A. R. 1989, Sol. Phys., 123, 217

Choudhuri, A. R. 1998, The physics of fluids and plasmas: an introduction for astrophysicists (Cambridge Univ. Press)

Choudhuri, A. R. 2003, Sol. Phys., 215, 31

Choudhuri, A. R., \& Gilman, P. A. 1987, ApJ, 316, 788

Choudhuri, A. R., Chatterjee, P., \& Nandy, D. 2004, ApJ, 615, L57

Choudhuri, A. R., Nandy, D., \& Chatterjee, P. 2005, A\&A, 437, 703

DeVore, C. R. 2000, ApJ, 539, 944

D'Silva, S. \& Choudhuri, A. R. 1993, A\&A, 272, 621

Fan, Y., \& Gong, D. 2000, Sol. Phys., 192, 141

Fan, Y., Fisher, G. H., \& Deluca, E. E. 1993, ApJ, 405, 390

Goldstein, S. 1938, Modern Developments in Fluid Mechanics (Oxford: Clarendon Press), p. 418

Hagino, M., \& Sakurai, T. 2005, PASJ, 57, 481

Hale, G. E. 1927, Nature, 119, 708

Holder, Z. A., Canfield, R. C., McMullen, R. A., et al. 2004, ApJ, 661, 1149

Hughes, S. J., Rajaguru, S. P., \& Thompson, M. J. 2005, ApJ, 627, 1040

Kosovichev, A. G., Duvall, T. L., \& Scherrer, P. H. 2000, Sol. Phys., 192,159

Leka, K. D., \& Skumanich, A. 1999, Sol. Phys., 188, 3

Longcope, D., \& Choudhuri, A. R. 2002, Sol. Phys., 205, 63

Longcope, D. W., Fisher, G. H., \& Pevtsov, A. A. 1998, ApJ, 507, 417

Moreno-Insertis, F. 1983, A\&A, 122, 241

Moreno-Insertis, F. 1986, A\&A, 166, 291

Nandy, D., \& Choudhuri, A. R. 2002, Science, 296, 1671

Petrovay, K., \& Moreno-Insertis, F. 1997, ApJ, 485, 398

Petrovay, K., \& van Driel-Gesztelyi, L. 1997, Sol. Phys., 176, 249

Pevtsov, A. A., Canfield, R. C., \& Latushko, S. M. 2001, ApJ, 549, L261

Pevtsov, A. A., Canfield, R. C., \& Metcalf, T. R. 1995, ApJ, 440, L109

Proctor, M. R. E. \& Weiss, N. O. 1982, Rep. Prog. Phys., 45, 1317

Richardson, R. S. 1941, ApJ, 93, 24

Schlichting, H. 1979, Boundary Layer Theory (7th ed., New York: McGraw-Hill), p. 15

Seehafer, N. 1990, Sol. Phys., 125, 219

Seehafer, N., Gellert, M., Kuzanyan, K. M., \& Pipin, V. V. 2003, Adv. Space Res., 32, 1819

Steiner, O. 2003, in Turbulence, Waves, and Instabilities in the Solar Plasma, ed. R. Erdélyi, K. Petrovay, B. Roberts, \& M. Aschwanden, NATO Science Ser. II.124 (Dordrecht: Kluwer), 117

Unno, W., Kondo, M., \& Xiong, D. R. 1985, PASJ, 37, 235

van Driel-Gesztelyi, L., Démoulin, P., \& Mandrini, C. H. 2003, Adv. Space Res., 32, 1855 